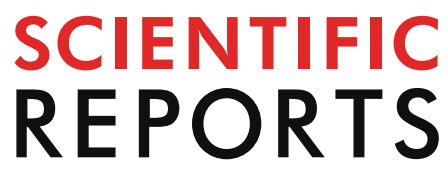

natureresearch

Check for updates

\title{
OPEN Greenbug (Schizaphis graminum) herbivory significantly impacts protein and phosphorylation abundance in switchgrass (Panicum virgatum)
}

Prince Zogli ${ }^{1,6}$, Sophie Alvarez ${ }^{2,6}$, Michael J. Naldrett ${ }^{2,6}$, Nathan A. Palmer ${ }^{3,6}$, Kyle G. Koch ${ }^{1}$, Lise Pingault ${ }^{1}$, Jeffrey D. Bradshaw ${ }^{1}$, Paul Twigg ${ }^{4}$, Tiffany M. Heng-Moss ${ }^{1}$, Joe Louis ${ }^{1,5 \bowtie}$ \& Gautam Sarath ${ }^{1,3 凶}$

Switchgrass (Panicum virgatum L.) is an important crop for biofuel production but it also serves as host for greenbugs (Schizaphis graminum Rondani; GB). Although transcriptomic studies have been done to infer the molecular mechanisms of plant defense against GB, little is known about the effect of GB infestation on the switchgrass protein expression and phosphorylation regulation. The global response of the switchgrass cultivar Summer proteome and phosphoproteome was monitored by label-free proteomics shotgun in GB-infested and uninfested control plants at 10 days post infestation. Peptides matching a total of 3,594 proteins were identified and 429 were differentially expressed proteins in GB-infested plants relative to uninfested control plants. Among these, 291 and 138 were up and downregulated by GB infestation, respectively. Phosphoproteome analysis identified 310 differentially phosphorylated proteins (DP) from 350 phosphopeptides with a total of 399 phosphorylated sites. These phosphopeptides had more serine phosphorylated residues $(79 \%)$, compared to threonine phosphorylated sites (21\%). Overall, KEGG pathway analysis revealed that GB feeding led to the enriched accumulation of proteins important for biosynthesis of plant defense secondary metabolites and repressed the accumulation of proteins involved in photosynthesis. Interestingly, defense modulators such as terpene synthase, papain-like cysteine protease, serine carboxypeptidase, and lipoxygenase2 were upregulated at the proteome level, corroborating previously published transcriptomic data.

Cereal aphids can successfully colonize and damage switchgrass (Panicum virgatum) plants ${ }^{1}$. Among the aphids tested, greenbugs (Schizaphis graminum, GB) caused significant plant damage likely through a combination of aphid-salivary proteins that are injected into plants during feeding and a strong host response elicited by herbivory ${ }^{2,3}$.

Plant resistance to herbivory has been broadly classified as antixenosis (deters insect settling), antibiosis (curtails insect fecundity), and tolerance ${ }^{4,5}$. Tolerant plants have compensatory mechanisms that allow for continued plant development with minimal yield losses, yet do not affect the fitness of the aphid ${ }^{6}$. Tolerance provides a reasonable means for improving plants in the face of continued pest pressure and are unlikely to select for the development of more virulent or resistant insect biotypes ${ }^{4}$.

\footnotetext{
${ }^{1}$ Department of Entomology, University of Nebraska-Lincoln, Lincoln, NE 68583-0816, USA. ${ }^{2}$ Proteomics and Metabolomics Facility, Nebraska Center for Biotechnology, University of Nebraska-Lincoln, Lincoln, NE 68588, USA. ${ }^{3}$ Wheat, Sorghum, and Forage Research Unit, USDA-ARS, Lincoln, NE 68583-0937, USA. ${ }^{4}$ Biology Department, University of Nebraska-Kearney, Kearney, NE 68849, USA. ${ }^{5}$ Department of Biochemistry, University of Nebraska-Lincoln, Lincoln, NE 68583-0816, USA. ${ }^{6}$ These authors contributed equally: Prince Zogli, Sophie Alvarez, Michael J. Naldrett and Nathan A. Palmer. ${ }^{\circledR}$ email: joelouis@unl.edu; Gautam.Sarath@ars.usda.gov
} 


\begin{tabular}{|l|l|l|l|}
\hline & Significant loci/proteins identified & Upregulated & Downregulated \\
\hline DEPs & 429 & 291 & 138 \\
\hline DEGs $^{\mathrm{a}}$ & 10,032 & 6,174 & 3,858 \\
\hline
\end{tabular}

Table 1. Total number loci identified and analyzed for this study. DEPs differentially expressed proteins, DEGs differentially expressed genes. ${ }^{\text {a Previously published work }}{ }^{3}$.

An evaluation of switchgrass plants for categories of resistance indicated that plants of upland cultivar Summer were moderately susceptible to GB herbivory, but also demonstrated responses consistent with tolerance ${ }^{7}$; however, GB could not colonize or inflict significant damage on the lowland cultivar Kanlow ${ }^{8,9}$. Subsequently, a time course study of the changes in select metabolites and the transcriptomes of Summer switchgrass plants infested with GB was performed ${ }^{3}$. In this study, it was demonstrated that plant defensive metabolites, such as pipecolic acid, chlorogenic acid, and reactive oxygen species (ROS) were induced in response to GB herbivory. There were significant alterations to the transcriptomes of the infested plants with a peak in transcriptional changes occurring 10 days post aphid infestation (10 DPI). Furthermore, consistent with data reported in the literature ${ }^{9,10}$, there was a significant downregulation of genes associated with nutrient assimilation, photosynthetic pigment biosynthesis, and other growth-related pathways, and a concomitant upregulation of genes involved in plant defense and catabolic processes.

ROS and ROS mitigation are critical processes determining host responses to aphid herbivory ${ }^{10,11}$. Initial ROS signals arise from the respiratory burst oxidases (RBOHs) present on the plasma membrane ${ }^{12}$. Subsequent signaling cascades involves both apoplastic and symplastic propagation. Excess ROS produced by the host are mitigated by several classes of enzymes, such as peroxidases (PRX), catalases (CAT), superoxide dismutases (SOD), and glutathione-S-transferases (GST) among others. A failure to mitigate excess ROS has been linked to susceptibility to aphid herbivory in several plant species ${ }^{13,14}$. Upregulation of peroxidase genes and protein activity have been frequently linked to aphid herbivory as well as to tolerant or resistant plants ${ }^{15}$.

Several proteomic studies examining insect-plant interactions ${ }^{16-21}$ have been reported in the literature. Earlier studies had used a combination of 2-dimensional gel electrophoresis (2DGE) followed by mass spectrometry (MS), and some of the more recent reports have used other approaches such as tandem mass tag (TMT) of proteins followed by MS/MS ${ }^{22,23}$. The general trend shown in the literature suggests that besides upregulation of stress responsive proteins, insect feeding leads to changes in plant metabolism (carbohydrate, amino acid, and energy metabolism) and photosynthesis. As a consequence, genes involved in secondary metabolite biosynthesis and photosynthesis are up and downregulated, respectively ${ }^{16,17}$. Furthermore, increased oxidative stress response is accompanied by upregulation of proteins involved in detoxification ${ }^{21}$. Other studies reported high accumulation of enzymes involved in jasmonic acid (JA) and ethylene biosynthetic pathways, as well as serine proteases/ protease inhibitors, in response to root herbivory ${ }^{24}$.

In this study, shotgun label-free proteomics ${ }^{25}$ has been used to document changes to the switchgrass proteome as a result of GB infestation. Additionally, changes in protein phosphorylation present in GB-infested and uninfested control plants was also determined. These proteomic data were compared against transcriptome changes recently published for this system ${ }^{3}$.

\section{Results}

Identification of differentially expressed proteins (DEPs). To investigate the mechanisms of switchgrass responses against GB, the proteomic profiles of aphid-infested and uninfested control plants was performed on 10 DPI (Fig. S1). This time was chosen based on earlier data showing that peak transcriptomic responses occurred $10 \mathrm{DPI}$, with subsequent dampening in the response at $15 \mathrm{DPI}^{3}$. Our goal was to capture as much differential details about the proteomes obtained from GB-infested and uninfested control plants and assess the extent to which GB infestation impacted the switchgrass proteome.

Peptide data generated in this study was used to search the switchgrass genome version 5.1 proteome database (https://phytozome-next.jgi.doe.gov/) and identified 3,594 proteins (with at least two unique peptides with high confidence, at $1 \%$ false discovery rate, Table S1). Overall, 429 of these proteins were identified as differentially expressed proteins (DEPs) with a $\log _{2}$ fold change Infected/Control $\left(\log _{2} \mathrm{FC}(\mathrm{I} 10 / \mathrm{C} 10)\right)$ that are significantly different based on an adjusted $p$ value $\leq 0.05$ cutoff criteria (Table S2). A total of 291 and 138 of the 429 DEPs were up and downregulated, respectively, in response to GB herbivory (Table 1). The numbers of differentially expressed genes (DEGs) and the corresponding up and downregulated genes obtained from a previous transcriptomic study ${ }^{3}$ are shown for comparison in Tables 1 and S2.

GB infestation leads to upregulation of proteins involved in oxidative and secondary metabolic pathways, but suppresses proteins involved in photosynthesis and other related pathways. Enrichment analyses with $\mathrm{GOBU}^{26}$ revealed that upregulated proteins were significantly enriched with several functions related to chitinase activity and biosynthesis of secondary metabolites (Table 2). Proteins associated with secondary plant metabolism included several peroxidases, $\beta$-glucosidase family 13 proteins, cytochrome P450 proteins (Pavir.5KG587200, a homolog of AT2G40890 involved in lignin and flavonoid biosynthesis), a S-adenosyl-L-methionine-dependent cinnamyl-CoA-O-methyltransferase (CCoAOMT1, Pavir.6KG340400), and phenylalanine ammonia lyase 1 proteins (Table S2). Among other DEPs were two NAD(P)-binding Rossmann proteins, Pavir.7KG263500 and Pavir.7KG263200, whose homologs have been 
GO terms significantly enriched

Enrichment analysis of upregulated DEPs

Catalytic activity

Molecular function

Biological process

Metabolic process

Hydrolase activity

Oxidoreductase activity

Oxidation-reduction process

Hydrolase activity, hydrolyzing O-glycosyl compounds

Hydrolase activity, acting on glycosyl bonds

Cofactor binding

Carbohydrate metabolic process

Response to oxidative stress

Heme binding

Tetrapyrrole binding

Antioxidant activity

Response to stress

Binding

Chitinase activity

Metal ion binding

Cation binding

Glucosamine-containing compound catabolic process

Glucosamine-containing compound metabolic process

Amino sugar metabolic process

Aminoglycan catabolic process

Chitin catabolic process

Chitin metabolic process

Amino sugar catabolic process

Peroxidase activity

Oxidoreductase activity, acting on peroxide as acceptor

Drug catabolic process

Cell wall macromolecule catabolic process

Cell wall macromolecule metabolic process

Aminoglycan metabolic process

Oxidoreductase activity, acting on single donors with incorporation of molecular oxygen, incorporation of two atoms of oxygen

Dioxygenase activity

Carbohydrate derivative catabolic process

Response to stimulus

Oxidoreductase activity, acting on single donors with incorporation of molecular oxygen

Peptidase activity

Exopeptidase activity

Proteolysis

Magnesium ion binding

Lyase activity

Hydrolase activity, acting on acid phosphorus-nitrogen bonds

Serine-type peptidase activity

Serine hydrolase activity

Serine-type carboxypeptidase activity

Carboxypeptidase activity

Peptidase activity, acting on L-amino acid peptides

Terpene synthase activity

Cell wall organization or biogenesis

Serine-type exopeptidase activity

Carbon-oxygen lyase activity, acting on phosphates

Ion binding

Continued
14,617

27,777

19,951

15,661

4,253

3,021

2,547

730

814

1,827

1,098

336

961

964

365

1,055

16,455

37
3,558

3,586

37

37

37

37

37

37

37

339

343

41

41

46

46

52

55

55

1,790

70

1,134

152

1,223

238

431

348

348

348

111

113

1,105

114

172

120

121

8,249

\begin{tabular}{|r|r|}
\hline 146 \\
\hline 190 \\
\hline 125 \\
\hline 59 \\
\hline 46 \\
\hline 42 \\
\hline 22 \\
\hline 23 \\
\hline 29 \\
\hline 20 \\
11 \\
17
\end{tabular}

3.63E-35 $1.19 \mathrm{E}-32$

$1.35 \mathrm{E}-26 \quad 4.44 \mathrm{E}-24$

\begin{tabular}{l|l}
$3.98 \mathrm{E}-21$ & $2.22 \mathrm{E}-18$
\end{tabular}

$5.41 \mathrm{E}-20 \quad 3.02 \mathrm{E}-17$

$5.30 \mathrm{E}-19 \quad 1.74 \mathrm{E}-16$

2.33E-16 $7.67 \mathrm{E}-14$

$3.60 \mathrm{E}-16 \quad 2.01 \mathrm{E}-13$

$5.84 \mathrm{E}-14 \quad 1.92 \mathrm{E}-11$

5.88E-14 $1.93 \mathrm{E}-11$

4.53E-11 $1.49 \mathrm{E}-08$

5.45E-09 $3.05 \mathrm{E}-06$

5.43E-08 $3.04 \mathrm{E}-05$

$1.18 \mathrm{E}-07 \quad 3.88 \mathrm{E}-05$

1.23E-07 $4.05 \mathrm{E}-05$

$1.25 \mathrm{E}-07 \quad 4.11 \mathrm{E}-05$

8.49E-08 $4.75 \mathrm{E}-05$

2.12E-07 $6.97 \mathrm{E}-05$

2.40E-07 $7.90 \mathrm{E}-05$

3.20E-07 0.000105

\begin{tabular}{l|l}
$3.82 \mathrm{E}-07$ & 0.000126
\end{tabular}

\begin{tabular}{l|l}
$2.40 \mathrm{E}-07$ & 0.000134
\end{tabular}

2.40E-07 0.000134

2.40E-07 0.000134

2.40E-07 0.000134

2.40E-07 0.000134

2.40E-07 0.000134

\begin{tabular}{l|l}
$2.40 \mathrm{E}-07$ & 0.000134
\end{tabular}

\begin{tabular}{l|l}
$5.69 \mathrm{E}-07$ & 0.000187
\end{tabular}

\begin{tabular}{l|l}
$6.33 \mathrm{E}-07$ & 0.000208
\end{tabular}

\begin{tabular}{l|l}
$4.07 \mathrm{E}-07$ & 0.000228
\end{tabular}

\begin{tabular}{l|l}
$4.07 \mathrm{E}-07$ & 0.000228
\end{tabular}

\begin{tabular}{l|l}
$7.34 \mathrm{E}-07$ & 0.000410
\end{tabular}

\begin{tabular}{l|l}
$7.34 \mathrm{E}-07$ & 0.000410
\end{tabular}

\begin{tabular}{l|l}
$1.37 \mathrm{E}-06$ & 0.000451
\end{tabular}

\begin{tabular}{l|l}
$1.81 \mathrm{E}-06$ & 0.000595
\end{tabular}

\begin{tabular}{l|l}
$1.81 \mathrm{E}-06$ & 0.001012
\end{tabular}

3.13E-06 0.001750

\begin{tabular}{l|l}
$6.04 \mathrm{E}-06$ & 0.001987
\end{tabular}

\begin{tabular}{l|l}
$2.12 \mathrm{E}-05$ & 0.006975
\end{tabular}

$2.14 \mathrm{E}-05 \quad 0.007041$

\begin{tabular}{l|l}
$1.29 \mathrm{E}-05$ & 0.007211
\end{tabular}

2.94E-05 0.009673

\begin{tabular}{l|l}
$3.28 \mathrm{E}-05$ & 0.010791
\end{tabular}

4.60E-05 0.015134

4.60E-05 0.015134

4.60E-05 0.015134

\begin{tabular}{l|l}
$5.66 \mathrm{E}-05$ & 0.018621
\end{tabular}

$\begin{array}{ll}6.16 \mathrm{E}-05 & 0.020266\end{array}$

6.30E-05 0.020727

6.43E-05 0.021155

\begin{tabular}{ll|l}
$4.29 \mathrm{E}-05$ & 0.023981
\end{tabular}

\begin{tabular}{l|l}
$8.20 \mathrm{E}-05$ & 0.026978
\end{tabular}

\begin{tabular}{l|l}
$8.53 \mathrm{E}-05$ & 0.028064
\end{tabular}

\begin{tabular}{l|l}
0.00011 & 0.036848
\end{tabular} 


\begin{tabular}{|l|c|c|l|l|l|}
\hline GO terms significantly enriched & \# of GO terms present in reference genome & \# of GO term hits among DEPs & \multicolumn{1}{l|}{$\boldsymbol{p}$ value } & Adjusted $\boldsymbol{p}$ value \\
\hline Enrichment analysis of downregulated DEPs & \multicolumn{1}{|l|}{} \\
\hline Photosynthesis, light harvesting & 32 & 7 & $1.23 \mathrm{E}-13$ & $6.88 \mathrm{E}-11$ \\
\hline Catalytic activity & 14,617 & 61 & $1.75 \mathrm{E}-12$ & $5.76 \mathrm{E}-10$ \\
\hline Photosynthesis, light reaction & 56 & 7 & $8.22 \mathrm{E}-12$ & $4.59 \mathrm{E}-09$ \\
\hline Biological process & 19,951 & 71 & $1.75 \mathrm{E}-11$ & $9.78 \mathrm{E}-09$ \\
\hline Photosynthesis & 154 & 8 & $3.28 \mathrm{E}-10$ & $1.83 \mathrm{E}-07$ \\
\hline Generation of precursor metabolites and energy & 233 & 9 & $3.47 \mathrm{E}-10$ & $1.94 \mathrm{E}-07$ \\
\hline Metabolic process & 15,661 & 57 & $3.45 \mathrm{E}-09$ & $1.93 \mathrm{E}-06$ \\
\hline Carbohydrate metabolic process & 1,098 & 12 & $5.08 \mathrm{E}-07$ & 0.000284 \\
\hline Molecular function & 27,777 & 75 & $1.57 \mathrm{E}-06$ & 0.000517 \\
\hline Protein folding & 193 & 6 & $1.18 \mathrm{E}-06$ & 0.000660 \\
\hline Oxidoreductase activity & 3,021 & 18 & $4.77 \mathrm{E}-06$ & 0.001569 \\
\hline Hydrolase activity, hydrolyzing O-glycosyl compounds & 730 & 8 & $4.23 \mathrm{E}-05$ & 0.013917 \\
\hline Protein import & 36 & 3 & $3.40 \mathrm{E}-05$ & 0.019006 \\
\hline Oxidation-reduction process & 2,547 & 15 & $3.56 \mathrm{E}-05$ & 0.019900 \\
\hline Hydrolase activity, acting on glycosyl bonds & 814 & 8 & $9.01 \mathrm{E}-05$ & 0.029643 \\
\hline
\end{tabular}

Table 2. Enrichment analysis of significantly enriched PFAM domains, KEGG metabolic pathways, and molecular function GO terms among differentially expressed proteins (DEPs). Significant GO terms are reported here.

implicated in biosynthesis of defense-related terpenoids ${ }^{27}$. Similarly, three upregulated DEPs, Pavir.4KG114700, Pavir.9NG755900 and Pavir.6KG207900 were annotated as basic chitinases and homologs of Arabidopsis basic chitinase/PR3, which has been implicated as playing a role in the defense response of Arabidopsis ${ }^{28}$. Metabolic pathway enrichment also correlated well with biological process enrichment analysis and revealed an abundance of GO terms such as single-organism metabolism, oxidation-reduction process, response to stress, response to oxidative stress, and chitin catabolic/metabolic process (Table 2). Notably, protein domain analysis revealed a significant enrichment of NAD(P)-binding domain proteins and glutathione S-transferases (GST). The data included 14 upregulated NAD(P)-binding Rossman-fold proteins and 11 GSTs (Table S2), suggesting redox regulation as a critical component of switchgrass response to GB infestation. Arabidopsis homologs of three of the upregulated NAD(P) binding proteins, Pavir.9NG062049 (AT1G52340, ABA2), Pavir.7NG329400, (AT3G61220, SDR1) and Pavir.7KG263200 (AT2G24190, SDR2), are involved in resistance against microbial pathogens ${ }^{29,30}$.

Consistent with pathway enrichment, upregulated proteins were enriched with molecular functions GO terms associated with catalysis, oxidoreductase activity, daphnetin 3-O-glucosyltransferase activity, and flavonol 3-O-glucosyltransferase activity (Table 2). Downregulated proteins in GB-infested plants were significantly enriched with biological process GO terms associated with photosynthetic and metabolic pathways (Table 2). All the downregulated proteins, such as Pavir.4KG305900, Pavir.5KG468900, Pavir.2NG555700 and Pavir.6KG271600, implicated in photosynthesis are involved with the Photosystem I light harvesting complex. Several other proteins linked to chloroplast function, such as albino or glassy yellow1, phytoene desaturase, thioredoxins, and uroporphyrinogen-III synthase, suggest a loss in plastid functions. Proteins linked to sucrose metabolism, such as sucrose phosphate synthase (SPSS) and a protein phosphatase that can dephosphorylate SPSS (BRI1 suppressor 1-like 2), were downregulated, indicating changes in sucrose biosynthesis (Table S2). Though downregulated proteins were also enriched with proteins having catalytic activity, as seen for the upregulated protein set, there was enrichment in chlorophyll binding and pigment binding, categories not enriched in GBinduced up-regulated proteins The group of proteins implicated in catalytic activity for the upregulated DEPs in response to GB herbivory were Pavir.6KG340400: CCoAMT1; Pavir.9NG661700: ALD1; Pavir.9KG072900: ornithine-delta-aminotransferase; and Pavir.3NG211100: terpenoid cyclase and downregulated proteins were Pavir.1NG556800: cytochrome P450; Pavir.5NG345600: peroxidase family protein; and Pavir.1KG250105: terpene synthase, suggesting that aphid attack in switchgrass remodels switchgrass metabolism.

Identification of phosphorylated sites and their abundance changes. Protein phosphorylation is important for plant defense signaling ${ }^{31,32}$. To explore the roles of protein phosphorylation in switchgrass defense signaling, the phosphoproteome of switchgrass at 10 DPI was profiled using LC-MS/MS after phosphoenrichment of the same protein extracts used for proteomics analysis. A total of 2,044 phosphopeptides matching 996 proteins were identified (Table S3) with high confidence $(<1 \%$ peptide false discovery rate). Amongst these phosphopetides identified, 1,786 of them carried a single phosphorylation, 229 with two phosphorylation and 29 with three or more phosphorylation. The overlap in phosphosites between phosphopeptides is shown in Fig. S2. The number of unique phosphosites from the singly, doubly or triply and more phosphorylated peptides was $1,455,398$ and 74, respectively (Fig. S2). The total number of unique phosphosites identified and quantified here is 1,779 . Amongst the 996 phosphoproteins, 310 were identified with a differentially phosphorylated (DP) site at 10 DPI with GB using following restrictions: (1) phosphopeptide detected in all four biological replicates, (2) adjusted $p$ value $<0.05$ and (3) $\mid \log _{2}$ FC (I10/C10) $\mid>1$ (Table S4). Because of the allotetraploid nature of the switchgrass genome, if a phosphopeptide is associated with two or more potentially homeologous genes, all 
A

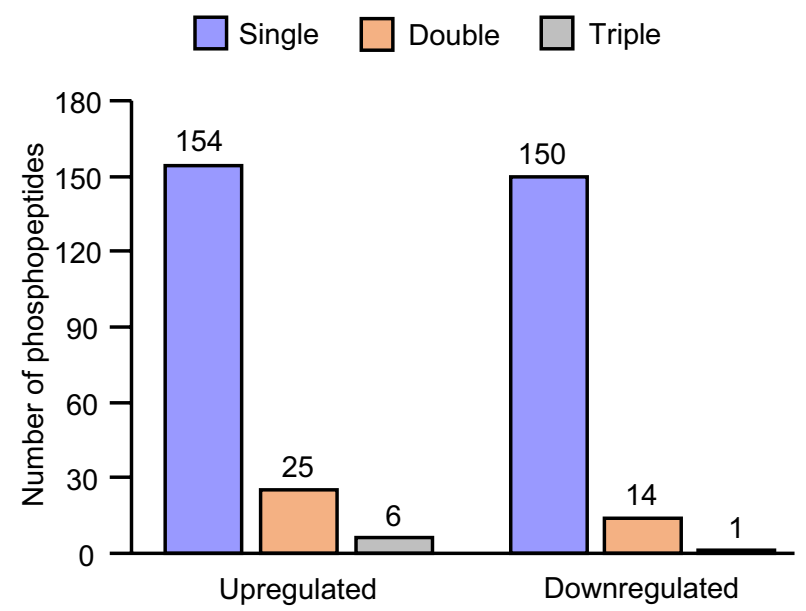

B
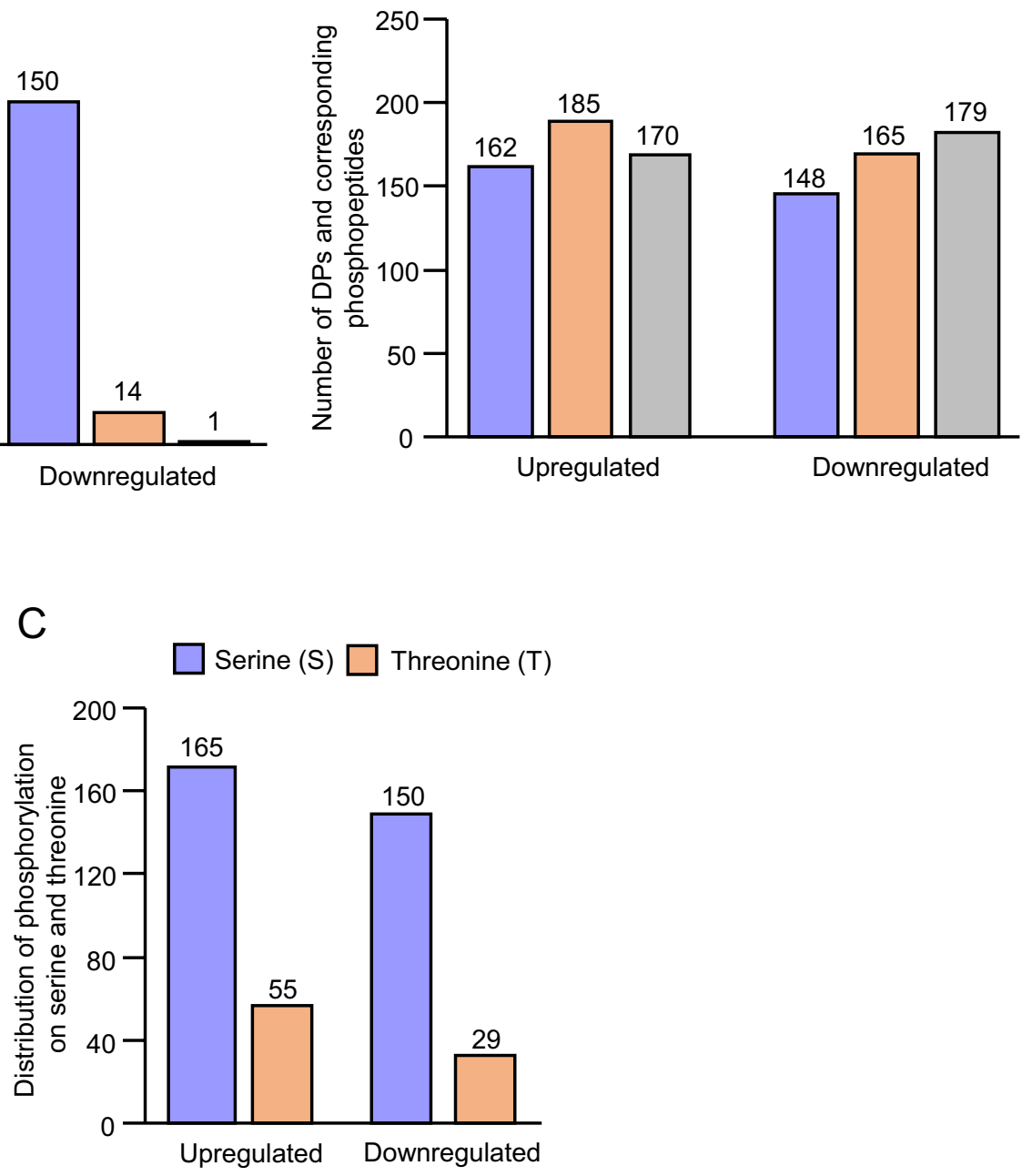

Figure 1. LC-MS/MS identification for phosphorylation sites of differentially phosphorylated proteins (DPs) from greenbug (GB)-infested switchgrass leaves. Leaf tissues were collected after 10 days post infestation of GB on switchgrass. (A) Distribution of single- and multi-phosphorylated peptides among DPs. (B) Number of phosphoproteins, phosphopeptides and phosphosites identified in the analysis. (C) The distribution of phosphorylated residues on serine $(\mathrm{S})$ and threonine $(\mathrm{T})$.

genes associated with significant phosphopeptides have been used for the analysis. This implies that a same phosphopeptide can be present more than once in the data set. These 310 DPs were identified with 350 phosphopeptides, which in total had 399 phosphosites (Table S5).

Figure 1A shows the distribution of the phosphopeptides up and downregulated identified with one, two or three phosphosites. About $87 \%$ of the phosphopeptides only have one phosphosite and $87 \%$ of the proteins with only one phosphopetide identified as DP. Among the 350 phosphopeptides, 185 had a significant increase in phosphorylation level, while 165 had a significant decrease in phosphorylation level in response to GB herbivory (Fig. 1B). The phosphorylated sites associated with the DPs are represented by 315 phosphoserines (79\%) and 84 phosphothreonines (21\%) (Fig. 1C). Interestingly, we identified a subset of 25 unique phosphopeptides associated with 12 proteins that displayed opposite phosphorylation abundance in response to GB herbivory (Table S6), which shows the importance of studying phosphorylation changes at each single phosphosite/phosphopeptide and not at the protein level. For example, among the three unique peptides associated Pavir.1KG036500, two had a significant increase in phosphorylation level while the other had a significant decrease in phosphorylation level (Table S6). Also, a cellulose synthase (Pavir.2NG127200) was identified with two peptides phosphorylated at positions S9 and S13 that were dephosphorylated and phosphorylated respectively, in response to GB herbivory. Other proteins with similar pattern of differentially phosphorylated or dephosphorylated residues included two ubiquitin-specific protease C19-related proteins (Pavir.6KG188500 and Pavir.6NG196600), two IQ-domain 14 proteins (Pavir.5KG696000 and Pavir.5NG012315), a RPM1 interacting protein4 (RIN4, Pavir.7KG167100), a hydroxyproline-rich glycoprotein (Pavir.2NG424700) and a calcium-binding EF hand protein (Pavir.4KG384303) 
(Table S6). These proteins may have very specific patterns of phosphorylation acting as switches in the regulation of defense mechanisms in switchgrass.

Changes to the subcellular partitioning of proteins is an essential part of plant defense responses, as different proteins need to be shuttled to specific cellular compartments to enact their functions, for example, cell wall fortification, redirection of primary metabolism, induction of gene expression, and protection of organelles from toxic byproducts to name just a few. In non-model plants such as switchgrass, these analyses also provide a means to comparing similar datasets with other more well characterized systems. BUSCA-based analysis (https://busca .biocomp.unibo.it/) showed that $63 \%, 10 \%, 8 \%, 8 \%$, and $5 \%$ of the 310 DPs were located in the nucleus, chloroplast, endomembrane system, plasma/organelle membrane, and cytoplasm, respectively (Fig. 2A). The remaining $6 \%$ were shared equally between mitochondria and the extracellular space (Fig. 2A). Similarly, 26\%, 22\%, 19\%, $14 \%, 7 \%, 5 \%, 4 \%$, and $3 \%$, of the 429 DEPs were located in the nucleus, chloroplast, plasma membrane, organelle membrane, endomembrane system, extracellular space, mitochondria, and cytoplasm, respectively (Fig. 2B).

\section{The multi-omics analysis shows correlations between gene regulation, protein abundance and phosphorylation changes in switchgrass upon GB infestation. A previous study at the transcrip-} tomic level showed that GB infestation activates cellular oxidative responsive pathways and suppresses photosynthesis and other related pathways ${ }^{3}$. As we observed a similar trend at the proteomic level in the current study, the proteomic data was compared to the 10,032 differentially expressed genes (DEGs) reported previously ${ }^{3}$. These included genes that were both up and downregulated as a consequence of GB herbivory ${ }^{3}$. Out of 6,018 upregulated DEGs, 114 were also detected as upregulated DEPs, while 31 out of the 3,858 downregulated DEGs were reported as downregulated DEPs (Tables 3 and S7, Fig. S3A).

Consistently, the group of genes upregulated at both transcript and proteome levels were enriched with proteins involved in secondary metabolite biosynthesis and stress/defense responses. These categories included Pavir.9NG661700 (ALD1), which is required for the biosynthesis of pipecolic acid, a key defensive compound that is significantly enhanced in response to GB herbivory, Pavir.6NG264600 (CCoAMT1) which participates in the biosynthesis of phenylpropanoid intermediates, Pavir.6KG367200 (LOX2) involved in oxylipin biosynthesis, Pavir.6NG135600 a mono/ sesquiterpene synthase that responds to herbivory ${ }^{33}$, Pavir.5KG357900 a glutathione S-transferase that is homologous to AT1G10360 (ERD9) that has been implicated in drought and stress tolerance in Arabidopsis ${ }^{34}$, and Pavir.2NG156200 (cytochrome P450 family protein, CYP99A3 family) potentially involved in the biosynthesis of phytoalexins and is also part of grass-specific family of proteins. Other defense genes like basic chitinases, PR3 Pavir.4KG114700 (AT3G12500) and PR4, Pavir.8KG305700 (AT3G04720) were also upregulated at both transcript and proteome level. Similarly, the group of genes downregulated at both transcriptome and proteome levels in response to GB were enriched with proteins such as Pavir.4KG305900, Pavir.6KG271600 and Pavir.2NG555700, which are chlorophyll a/b binding proteins and are involved in photosynthesis.

Overall, 429 DEPs in response to GB herbivory were identified, of which four upregulated DEPs were significantly increased in phosphorylation, while one downregulated DEP was dephosphorylated (Fig. S3B). In comparison, 64 DEGs were identified as differentially phosphorylated proteins (DPs) in response to GB infestation (Table 3). Among them, 25-upregulated and 18-downregulated DEGs also showed an increase and decrease in phosphorylation levels, respectively, upon GB infestation. The remaining 21 DEGs show an opposite response as follows: eight were upregulated DEGs but with a decrease in phosphorylation, and 13 were downregulated DEGs but significantly phosphorylated in response to GB herbivory (Table 4).

The group of genes upregulated at both transcript and proteome levels which also showed an increase in phosphorylation level in response to GB-feeding included proteins involved in plant-pathogen interactions, such as Pavir.9KG014300 (RIN4), Pavir.7KG306200 and Pavir.7NG308700 (CPK5), Pavir.5KG466400 and Pavir.5NG472800 (MKK6), and Pavir.6NG271832 (Hsp81.4). Conversely, downregulated DEGs that were decreased in phosphorylation level were enriched in proteins such as: Pavir.3NG183492 (STN7), Pavir.6NG092300 (PSBR), Pavir.4NG215687 (PPC2), Pavir.3NG076904 (PPDK), and Pavir.8NG194400 (LHCB5), which are involved in photosynthesis. A total of 13 transcription factors (TFs) were identified in this study only as DPs because these low abundance proteins, often regulated by phosphorylation, were enriched by the phosphoenrichment step in the phosphoproteomics study. Among the 13 TFs, two zinc-finger homeodomain proteins (Pavir.3NG065800 and Pavir.6KG276700) and a bZIP factor (Pavir.6KG394700), were transcriptionally up and downregulated respectively, in response to GB infestation. The remaining 10 TFs showed an increase in phosphorylation level only (Table S7), suggesting that these transcription factors are post-translationally regulated by phosphorylation in response to GB attack. These 10 TFs include a WRKY (Pavir.2NG560500), two calmodulin-binding transcription activators (Pavir.2KG546800, Pavir.9NG356600), one bHLH (MYC2; Pavir.9NG353828), and six zinc finger C3H TFs (Pavir.1NG017300, Pavir.2NG544400, Pavir.5NG172300, Pavir.7NG088447, Pavir.8KG143501 and Pavir.8NG213620).

\section{Discussion}

Proteomics has emerged as a powerful tool to explore physiological changes at the cellular level ${ }^{35,36}$ and has been used to study plant defense responses to herbivory ${ }^{19,22,23,37}$. The present study is a comprehensive analysis of changes occurring to the switchgrass proteome and phosphoproteome in response to GB herbivory. Switchgrass cultivars have distinct responses to aphids ${ }^{1}$, and the cultivar Summer, though damaged by GB appeared to have a tolerant response. Plant damage, physiological and transcriptomic studies of Summer switchgrass responses to GB infestation ${ }^{3}$ largely corroborated these earlier phenotypic findings indicating that 10 DPI is a good sampling time to assess changes in the switchgrass proteome as a result of GB infestation. To our knowledge, this is the first study that utilizes proteomic and phosphoproteomic approaches to monitor switchgrass defense responses to GB herbivory. The two methods identified a total of 3,594 proteins and 2,044 phosphorylated peptides belonging to 
A

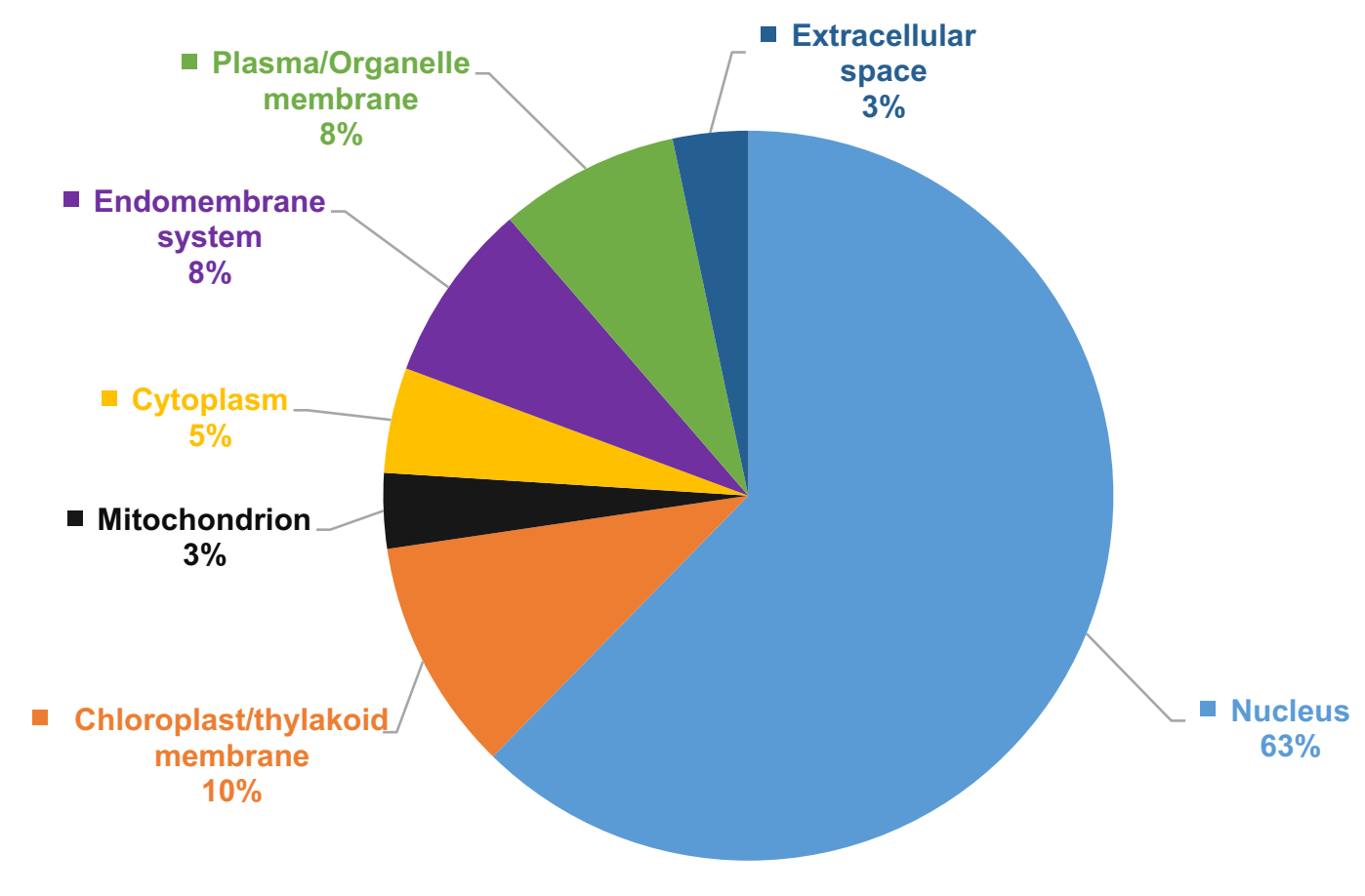

B

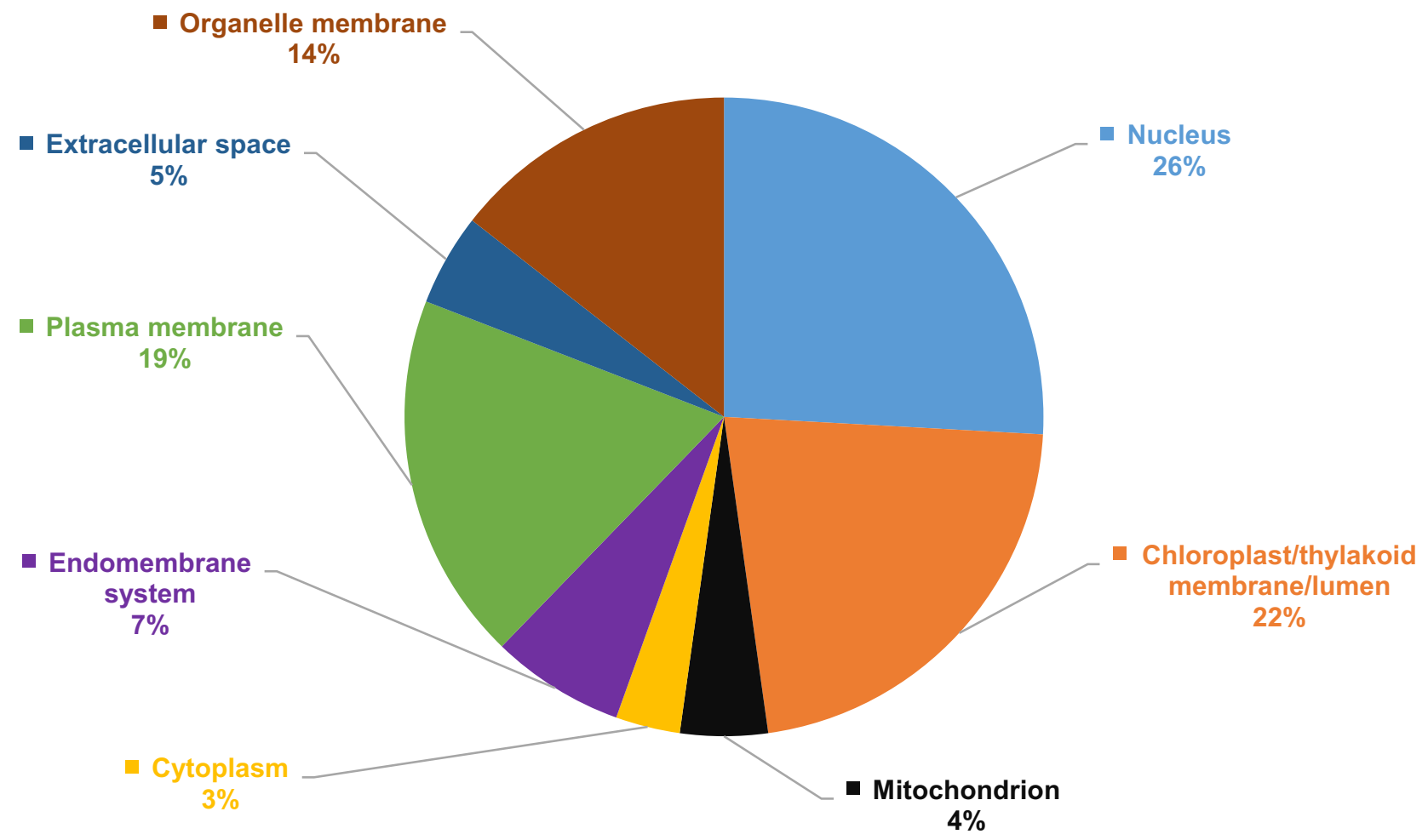

Figure 2. Subcellular localization of (A) DPs and (B) DEPs identified in switchgrass-greenbug interactions. BUSCA tool (https://busca.biocomp.unibo.it/) was used to analyze the subcellular localization of DPs and DEPs.

996 proteins. GO enrichment and KEGG pathway analysis indicated that proteins involved in host plant processes such as secondary metabolite metabolism, redox regulation, and photosynthesis are significantly altered by GB infestation. 


\begin{tabular}{|l|l|l|l|l|l|l|}
\hline & DEGs-up & & & & & \\
\hline DEGs-up & 6,018 & DEGs-down & & & & \\
\hline DEGs-down & & 3,777 & DEPs-up & & & \\
\hline DEPs-up & 114 & 19 & 154 & DEPs-down & & \\
\hline DEPs-down & 9 & 31 & & 97 & DPs-up & \\
\hline DPs-up & 25 & 13 & 4 & NA & 120 & DPs-down \\
\hline DPs-down & 8 & 18 & NA & 1 & & 120 \\
\hline
\end{tabular}

Table 3. Number of differentially expressed genes (DEGs), differentially expressed proteins (DEPs), and differentially phosphorylated proteins (DPs) identified in a comparative study.

DEPs involved in secondary metabolite biosynthesis. Secondary metabolites produced by plants can influence herbivore feeding on plant tissues ${ }^{38}$. Plants activate secondary metabolites as potential defense mechanisms against microbial and insect attacks. Several plant metabolites including alkaloids, terpenoids, isoflavonoids, oxoacids, carboxylic and benzoic acids negatively affects the performance of herbivores ${ }^{39}$. Oxoacid and carboxylic acid-dependent defense pathways against insects through glucosinolates and SA-dependent gene regulation were reported in the model plant Arabidopsis ${ }^{40,41}$. Evidence from maize indicates a role for the benzoxazinoid and related products of plant secondary metabolism in plant defense ${ }^{42,43}$. It is tempting to speculate that switchgrass would similarly synthesize secondary metabolites in response to insect stress. The present study uncovered differential regulation of several proteins involved in secondary metabolite biosynthesis. Proteins induced by GB infestation included enzymes essential for the biosynthesis of phenylpropanoid intermediates (CCoAMT1, Pavir.6NG264600) ${ }^{44}$, terpene biosynthesis, glutathione-related metabolism, and oxylipin biosynthesis. Elevated levels of terpene synthases are consistent with recent transcriptomic and biochemical data that indicated a significant upregulation of switchgrass terpene synthase encoding genes and increased enzymatic activities in response to feeding by aphids and caterpillars ${ }^{3,33}$. However, not all genes associated with specific aspects of secondary plant metabolism were induced by insect herbivory, suggesting role(s) in other aspects of plant metabolism.

Four lipoxygenases (LOXs), encoded by Pavir.6KG367200, Pavir.1KG101800 Pavir.6NG315500 and Pavir.3KG264209, were induced in response to GB. LOXs catalyze the oxidation of polyunsaturated fatty acids generating hydroperoxy fatty acids. The Arabidopsis homolog, AtLOX2 (AT3G45140) encodes a 13(S)-lipoxygenase that controls the first dedicated step in the biosynthesis of JA $\mathrm{A}^{45}$. JA is an important hormone induced in response to insect herbivory ${ }^{46}$. Therefore, the significant induction of LOX2 homologs suggest that switchgrass relies on LOX2-mediated JA signaling to activate their basal defense. In Arabidopsis, AtLOX2 is constitutively phosphorylated at $\mathrm{Ser}_{600}$ and targeted by insect salivary effectors for dephosphorylation to lower JA accumulation ${ }^{47}$, thereby promoting herbivory. The AtLOX2 homolog in switchgrass (Pavir.7KG108800) was significantly upregulated and phosphorylated in response to GB herbivory, which suggest a similar AtLOX2dependent JA signaling mechanism may exist in switchgrass to deter herbivory. However, a LOX family protein (Pavir.9NG150900), likely a LOX5 homolog, was downregulated in switchgrass after GB infestation. Root enhanced expression of Arabidopsis LOX5 positively impacts green peach aphid (Myzus persicae) feeding ${ }^{48}$. It is intriguing to speculate that downregulation of switchgrass LOX5 may have a negative influence on GB feeding. Taken together, the current proteomic, phosphoproteomic, and previous transcriptomic data ${ }^{3}$, indicate that JA biosynthesis is likely impacted via a complex regulatory network in switchgrass.

DEPs involved in oxidative stress. In response to plant herbivores, reactive oxygen species (ROS) are generated in plant tissues ${ }^{49}$. Plant defense responses against aphids include calcium influxes ${ }^{50}$, accumulation of ROS $^{51}$, phloem occlusion ${ }^{52,53}$, and callose deposition ${ }^{54}$. High and low accumulation or reduction of ROS make plants resistant or susceptible to aphids, respectively. For instance, the induction of ROS activity was very high in the resistant wheat (Triticum aestivum) infested with Russian wheat aphid (Diuraphis noxia), but low when infested with a more virulent $D$. noxia ${ }^{55}$. Also, GB feeding on resistant sorghum (Sorghum bicolor) genotype caused high expression of peroxidase leading to ROS production ${ }^{56}$. ROS is proposed to be produced by different enzymes, including NADPH oxidases ${ }^{57}$, peroxidase $^{58}$, and oxalate oxidase ${ }^{59}$. However, though ROS are important signaling molecules in plants, high abundance can be toxic to the plant and ROS scavenging is critical for plant health. As such, high amounts of ROS can be removed by ROS-scavenging enzymes like catalases ${ }^{60}$, peroxidases $^{61}$, and superoxide dismutases ${ }^{62}$, as well as antioxidants like ascorbate and glutathione ${ }^{63}$. Apart from scavenging, peroxidases are essential players in auxin catabolism, programmed cell death, defenses against pathogens, and cell wall lignification ${ }^{64}$. Oxidative stress-related proteins were differentially regulated upon GB infestation, and included peroxidases, catalases, superoxide dismutates, dioxygenases, and other reductases and GSTs. Interestingly, switchgrass oxidative stress proteins were upregulated in response to drought ${ }^{65}$, suggesting switchgrass may use similar pathways to combat biotic and abiotic stress. Pavir.8NG068900, a homolog of Arabidopsis $\alpha$-dioxygenases ( $\alpha$-DOX1, AT3G01420), is an enzyme that may contribute to the synthesis of oxylipin, a signaling molecule implicated in plant defense against tobacco hornworm and aphids ${ }^{66,67}$. It is highly likely that Pavir.8NG068900, like other a-DOXs, may play a similar role in conferring switchgrass resistance to GB herbivory. 


\begin{tabular}{|c|c|c|c|c|}
\hline Gene ID & Best hit Arabidopsis & Function & $\begin{array}{l}\text { Phospho-peptide sequence and phosphorylated } \\
\text { residues (in bold) }\end{array}$ & Category \\
\hline Pavir.1KG096400 & NA & NA & KVPSRPPSAHGHGHAPAPAPK & DEGs-up DPs-down \\
\hline Pavir.1KG181862 & AT1G53310 & Phosphoenolpyruvate carboxylase 1 & IRDPAFQVSPQPALSK & DEGs-up DPs-up \\
\hline Pavir.1KG219100 & AT2G36460 & Aldolase superfamily protein & ${ }^{1}$ GILAADESTGTIGKR & DEGs-up DPs-up \\
\hline Pavir.1KG438510 & AT1G20440 & Cold-regulated 47 & $\begin{array}{l}\text { EKLPGGHKKPEDAAAPAVHAPAPAPHAEDVG- } \\
\text { SPDGK }\end{array}$ & DEGs-up DPs-up \\
\hline Pavir.1KG456400 & AT4G13940 & S-adenosyl-L-homocysteine hydrolase & ${ }^{1}$ LTKSQADYISVPIEGPYKPAHYR & DEGs-up DPs-up \\
\hline Pavir.1NG430900 & AT4G13940 & S-adenosyl-L-homocysteine hydrolase & ${ }^{1}$ LTKSQADYISVPIEGPYKPAHYR & DEGs-up DPs-up \\
\hline Pavir.2KG411435 & AT2G33620 & AT hook motif DNA-binding family protein & VAPAAPSSPPSR & DEGs-up DPs-up \\
\hline Pavir.2KG484600 & AT4G05150 & Octicosapeptide/Phox/Bemlp family protein & SDAAETPRQHGDEDEASVPAR & DEGs-up DPs-down \\
\hline Pavir.2KG572300 & AT1G53050 & Protein kinase superfamily protein & IADFGLASFFDPNHKQPMTSR & DEGs-up DPs-up \\
\hline Pavir.2NG515300 & AT1G66950 & Pleiotropic drug resistance 11 & ${ }^{1}$ WAAIEKLPTYDR & DEGs-up DPs-up \\
\hline Pavir.2NG515300 & AT1G66950 & Pleiotropic drug resistance 11 & SWLSAASISR & DEGs-up DPs-up \\
\hline Pavir.3KG124322 & AT4G09160 & $\begin{array}{l}\text { Sec14 cytosolic factor family protein/phosphoglycer- } \\
\text { ide transfer family protein }\end{array}$ & $\begin{array}{l}\text { AAEADSEEEKKAEEALEAAAGDEAAVID- } \\
\text { GAGSFK }\end{array}$ & DEGs-up DPs-up \\
\hline Pavir.3KG261700 & AT4G15530 & Pyruvate orthophosphate dikinase & ${ }^{1}$ SDFEGIFR & DEG-down DPs-down \\
\hline Pavir.3KG310400 & AT1G75220 & Major facilitator superfamily protein & AGGAGYESGSDHDGALQKPLLPNSGSWYR & DEG-down DPs-down \\
\hline Pavir.3KG402302 & AT1G15140 & FAD/NAD(P)-binding oxidoreductase & VVQLTQQFQQSFLEQNLGEK & DEG-down DPs-up \\
\hline Pavir.3NG065800 & AT1G75240 & Homeobox protein 33 & $\begin{array}{l}\text { VHLVGDPEHLGQLGGGMPLPEPGGPGRSPSP- } \\
\text { SRSPPPQQLR }\end{array}$ & DEG-down DPs-up \\
\hline Pavir.3NG066600 & AT1G33800 & Protein of unknown function (DUF579) & SSSSPMHAR & DEG-down DPs-down \\
\hline Pavir.3NG066600 & AT1G33800 & Protein of unknown function (DUF579) & KAIHLASLR & DEG-down DPs-down \\
\hline Pavir.3NG076904 & AT4G15530 & Pyruvate orthophosphate dikinase & ${ }^{1}$ SDFEGIFR & DEG-down DPs-down \\
\hline Pavir.3NG183492 & AT1G68830 & STT7 homolog STN7 & IVTTIKESMDELTSQRK & DEG-down DPs-down \\
\hline Pavir.3NG197700 & AT1G15140 & FAD/NAD(P)-binding oxidoreductase & AVQTQGAGVQTQQGGAAR & DEG-down DPs-up \\
\hline Pavir.3NG205200 & AT3G48740 & Nodulin MtN3 family protein & LPTTAAADEHVLVNIAKLSPALPEK & DEG-down DPs-down \\
\hline Pavir.3NG212697 & AT3G16630 & $\begin{array}{l}\text { P-loop containing nucleoside triphosphate hydro- } \\
\text { lases superfamily protein }\end{array}$ & AQNSANTQEEEKVTKVSPPR & DEGs-up DPs-down \\
\hline Pavir.3NG233000 & AT2G38280 & $\begin{array}{l}\text { AMP deaminase, putative / myoadenylate deaminase, } \\
\text { putative }\end{array}$ & $\begin{array}{l}\text { VAVIRPNSPKSPAASASAFESVDGSDED- } \\
\text { DATQHGGK }\end{array}$ & DEG-down DPs-up \\
\hline Pavir.4KG382102 & AT2G16860 & GCIP-interacting family protein & RVVPAAADDSDEEAGAER & DEGs-up DPs-down \\
\hline Pavir.4NG215687 & AT2G42600 & Phosphoenolpyruvate carboxylase 2 & YGMSYIHETIWK & DEG-down DPs-down \\
\hline Pavir.4NG215687 & AT2G42600 & Phosphoenolpyruvate carboxylase 2 & DAGRLSAAWQLYR & DEG-down DPs-down \\
\hline Pavir.4NG215687 & AT2G42600 & Phosphoenolpyruvate carboxylase 2 & QLAPGKVSEDDKLVEYDVLLMER & DEG-down DPs-down \\
\hline Pavir.5KG412607 & AT1G15520 & Pleiotropic drug resistance 12 & ${ }^{1}$ WAAIEKLPTYDR & DEGs-up DPs-up \\
\hline Pavir.5KG453100 & AT5G14740 & Carbonic anhydrase 2 & ${ }^{1}$ LKSGFEQFK & DEG-down DPs-down \\
\hline Pavir.5KG453100 & AT5G14740 & Carbonic anhydrase 2 & 'ISGFEQFKTQVYDKKPELFEPLK & DEG-down DPs-down \\
\hline Pavir.5KG466400 & AT5G56580 & MKK6 & ${ }^{1}$ FLTASGTFK & DEGs-up DPs-up \\
\hline Pavir.5NG012556 & AT2G36460 & Aldolase superfamily protein & ${ }^{1}$ GILAADESTGTIGKR & DEGs-up DPs-up \\
\hline Pavir.5NG016600 & AT5G17380 & $\begin{array}{l}\text { Thiamine pyrophosphate dependent pyruvate decar- } \\
\text { boxylase family protein }\end{array}$ & ADSAASNPSPPNQKPLDEAIGK & DEGs-up DPs-up \\
\hline Pavir.5NG178500 & ATCG00710 & Photosystem II reaction center protein $\mathrm{H}$ & ATQTVEDSSRPKPK & DEG-down DPs-up \\
\hline Pavir.5NG263800 & AT5G14740 & Carbonic anhydrase 2 & ${ }^{1}$ LKSGFEQFK & DEG-down DPs-down \\
\hline Pavir.5NG263800 & AT5G14740 & Carbonic anhydrase 2 & ${ }^{1}$ SGFEQFKTQVYDKKPELFEPLK & DEG-down DPs-down \\
\hline Pavir.5NG472800 & AT5G56580 & MKK6 & ${ }^{1}$ FLTASGTFK & DEGs-up DPs-up \\
\hline Pavir.5NG577900 & AT5G60010 & $\begin{array}{l}\text { Ferric reductase-like transmembrane component } \\
\text { family protein }\end{array}$ & AQSPGAGAGAGAGAGAGR & DEG-down DPs-up \\
\hline Pavir.6KG023500 & AT3G04120 & $\begin{array}{l}\text { Glyceraldehyde-3-phosphate dehydrogenase c } \\
\text { subunit } 1\end{array}$ & HSDITLKDSKTLLFGEKPVTVFGIR & DEGs-up DPs-down \\
\hline Pavir.6KG076000 & AT1G34210 & Somatic embryogenesis receptor-like kinase 2 & LMDYKDTHVTTAVR & DEG-down DPs-up \\
\hline Pavir.6KG276700 & AT5G15210 & Homeobox protein 30 & VVHPGPSVASGADSPLSA & DEG-down DPs-down \\
\hline Pavir.6KG290606 & AT1G56220 & Dormancy/auxin associated family protein & KYASFSPSSSSSLAPAAAPAVTR & DEG-down DPs-up \\
\hline Pavir.6KG394700 & AT4G38900 & $\begin{array}{l}\text { Basic-leucine zipper (bzip) transcription factor fam- } \\
\text { ily protein }\end{array}$ & LNFAAGDESPKLPLPSPGGGLTR & DEGs-up DPs-up \\
\hline Pavir.6NG088100 & NA & NA & NLGYTYDAESEKELPWVASK & DEG-down DPs-down \\
\hline Pavir.6NG092300 & AT1G79040 & Photosystem II subunit R & IKTDKPFGIGGGLTVDHDASGRK & DEG-down DPs-down \\
\hline Pavir.6NG176100 & AT5G15490 & UDP-glucose 6-dehydrogenase family protein & DLAMNKFDWDHPMHLQPTSPSAVK & DEG-down DPs-down \\
\hline Pavir.6NG271832 & AT5G56000 & Heat shock protein 81.4 & TTEKEISDDEDEEDKK & DEGs-up DPs-up \\
\hline Pavir.7KG080900 & AT5G58140 & Phototropin 2 & EIVEEPASSSPGAAAAGGGSYRQPSFQR & DEG-down DPs-down \\
\hline Pavir.7KG080900 & AT5G58140 & Phototropin 2 & SGSGGGKEIVEEPASSSPGAAAAGGGSYR & DEG-down DPs-down \\
\hline \multicolumn{5}{|l|}{ Continued } \\
\hline
\end{tabular}




\begin{tabular}{|c|c|c|c|c|}
\hline Gene ID & Best hit Arabidopsis & Function & $\begin{array}{l}\text { Phospho-peptide sequence and phosphorylated } \\
\text { residues (in bold) }\end{array}$ & Category \\
\hline Pavir.7KG182509 & ATCG00120 & ATP synthase subunit alpha & EAIQEQLERFSLQEQT & DEG-down DPs-down \\
\hline Pavir.7KG182518 & ATCG00120 & ATP synthase subunit alpha & VINALAKPIDGRGEIVASESR & DEG-down DPs-down \\
\hline Pavir.7KG182671 & ATCG00020 & Photosystem II reaction center protein A & TAILER & DEG-down DPs-up \\
\hline Pavir.7KG306200 & AT4G35310 & Calmodulin-domain protein kinase 5 & HAASQRQDSEYSAADDSPKKPSTR & DEGs-up DPs-up \\
\hline Pavir.7NG308700 & AT4G35310 & Calmodulin-domain protein kinase 5 & AASQRQDSEYSAAAADDSPKKPASR & DEGs-up DPs-up \\
\hline Pavir.7NG390200 & AT2G18960 & $\mathrm{H}(+)$-ATPase 1 & GLDIDTIQQNYTV & DEGs-up DPs-up \\
\hline Pavir.7NG401100 & AT1G56080 & NA & VTPGSTPMISSTGGSPR & DEGs-up DPs-up \\
\hline Pavir.8NG016300 & AT1G33110 & Mate efflux family protein & SFISKDDDEQQVEEESSSLGR & DEGs-up DPs-up \\
\hline Pavir.8NG106056 & AT3G45780 & Phototropin 1 & RKSQEADCVFSTQVPGK & DEG-down DPs-up \\
\hline Pavir.8NG194400 & AT4G10340 & Light harvesting complex of photosystem II 5 & KPAQKPKPAAVSSSSPDISDELAK & DEG-down DPs-down \\
\hline Pavir.9KG014300 & AT3G25070 & RPM1 interacting protein 4 & SATQNDNKGDPETPSKDPPSAK & DEGs-up DPs-up \\
\hline Pavir.9KG084000 & AT5G04890 & Hsp20-like chaperones superfamily protein & RPSLPRKPSAVEPPAPELPAR & DEGs-up DPs-up \\
\hline Pavir.9KG385400 & AT1G13740 & ABI5 binding protein 2 & TLGSLTTR & DEG-down DPs-up \\
\hline Pavir.9KG394400 & AT1G60420 & DC1 domain-containing protein & FKVSGIPHLVILDAK & DEGs-up DPs-down \\
\hline Pavir.9KG412083 & AT1G59710 & Protein of unknown function (DUF569) & LESSDSFSAPLHK & DEGs-up DPs-up \\
\hline Pavir.9KG451600 & AT2G41740 & Villin 2 & AAAVAALSNVLTAEGSHSPHHGRGSPTADAAK & DEG-down DPs-up \\
\hline Pavir.9KG530300 & AT2G02000 & Glutamate decarboxylase 3 & AVSESDMSVHSTFASR & DEGs-up DPs-up \\
\hline Pavir.9NG048300 & AT3G29360 & UDP-glucose 6-dehydrogenase family protein & DLAMNKFDWDHPMHLQPTSPTAVK & DEGs-up DPs-down \\
\hline Pavir.9NG319500 & AT2G03440 & Nodulin-related protein 1 & ELEPVPAAEEGKSEGFGLDDLVK & DEG-down DPs-down \\
\hline Pavir.9NG472000 & AT4G17330 & G2484-1 protein & ASASPEQQSVIASPQLK & DEG-down DPs-up \\
\hline Pavir.9NG600700 & AT2G39050 & Hydroxyproline-rich glycoprotein family protein & ILPWGDEAYAGGSAANAPHGGHGHGEPTVR & DEGs-up DPs-down \\
\hline
\end{tabular}

Table 4. List of previously reported DEGs significantly phosphorylated in this study. NA indicate that the loci is not found in the reference dataset or category. ${ }^{1}$ Sequence represents items associated with more than one loci.

DEPs and DPs involved in stress response. Plants have constitutive and inducible protective mechanisms to overcome various biotic and abiotic stresses ${ }^{68}$. Pathogenesis-related proteins (PR proteins) and defenserelated proteins are specifically induced under stress conditions ${ }^{68}$. In this study, several known stress response proteins were induced in switchgrass in response to GB herbivory. Among them were papain family cysteine proteases (Pavir.1KG224700), PR1 (Pavir.5KG293200), PR4s (Pavir.8NG270602, Pavir.8KG305700), disease resistance-responsive protein (Pavir.3KG066327), MLP-like protein (Pavir.3NG236300), and HOPW1-1-interacting2 (Pavir.7KG429900 and Pavir.3NG149165) proteins. Papain-like cysteine protease (PLCPs) are increasingly being reported as key players in plant immune signaling pathways ${ }^{69}$. In fact, a papain-like cysteine protease, Maize insect resistance1-Cysteine Protease (Mir1-CP) has been shown to provide direct toxicity to corn leaf aphid (Rhopalosiphum maidis) ${ }^{70}$. Similarly, AtHOPW1-1-interacting proteins act as receptors to recognize Pseudomonas syringae effector HOPW 1-1, leading to disease resistance in Arabidopsis ${ }^{71}$. It is therefore possible that Pavir.7KG429900 functions as a similar receptor for aphid-secreted effectors to activate resistance. However, another member of HOPW1-1-interacting protein family (Pavir.1NG023600) was downregulated after GB infestation, suggesting that effectors present in GB saliva may have evolved strategies to repress the function of this protein.

When comparing phosphoproteomic data generated in this study to previously published RNA-seq data ${ }^{3}$, 64 DPs were similarly regulated at the transcript level. Among those, 25 were significantly upregulated at the transcription and phosphorylation levels and phosphorylated in response to GB feeding (Table 4; see DEGsup DPs-up category). These groups of proteins were enriched in proteins involved in plant-pathogen interaction pathway, including: Pavir.6NG271832 (heat shock protein), Pavir.6KG394700 (bZIP transcription factor), Pavir.5NG472800 (MAP kinase kinase6), Pavir.7KG306200 and Pavir.7NG308700 (homeologous pair calmodulin-domain protein kinases), and Pavir.9KG014300 (RPM1 interacting protein 4, RIN4). Arabidopsis RIN4 and its orthologs are conserved in land plants and are targeted and phosphorylated by $P$. syringae secreted virulence proteins ${ }^{72}$. Intriguingly, RIN4 phosphorylation and its specific protein-protein interaction can activate or suppress plant immune responses ${ }^{72}$. For example, AvrRpm1 was recently reported to act as ADP-ribosyl transferase that promotes phosphorylation of AtRIN4, thereby inhibiting secretion of defense compounds $s^{73}$. Though AtRIN4 is phosphorylated at Thr-21, 166, and S20, Pavir.9KG014300 (PvRIN4) identified in this study is phosphorylated at S54, suggesting that RIN4 may be regulated differently in herbivory defense compared to defense against bacteria. Therefore, it will be interesting to know whether switchgrass RIN4's phosphorylation is also a mechanism utilized by aphid effectors to enhance virulence. Two uncharacterized DPs, Pavir.7NG401100 (NAI2-Interacting Protein 3, NAIP3) and Pavir.9KG412083 (actin cross-linking protein), were also reported as DEGs. Recent reports implicate NAIPs in the biogenesis of ER Bodies ${ }^{74}$. Because ER bodies are also known for providing defense against herbivor ${ }^{75,76}$, further investigation of the role of Pavir.7NG401100 in switchgrass defense to aphids is intriguing. 


\section{Conclusions}

In the present study, the proteomic and phosphoproteomic responses of switchgrass cultivar Summer to GB attack at 10 DPI was conducted. Previous research suggested that plant metabolism is altered during insect attack. In this study, we observed a global repression of photosynthesis, but upregulation of pathways involved in secondary metabolite biosynthesis. Besides changes in secondary metabolite biosynthesis, herbivory leads to numerous changes in plant primary metabolism as well ${ }^{77}$. Repression of photosynthesis is among the early responses to aphid herbivory, and the proteomic data are consistent with transcriptomic data published previously for switchgrass-GB interactions ${ }^{3}$. This suggest suppression of photosynthesis is a global response to biotic stress attacks, potentially to reduce the amount of nutrients available to the herbivore, redirect the movement of sucrose to distal sources, and recalibrate the sugars to starch ratios.

In addition, we found some correlation between regulation of protein abundance and protein phosphorylation in response to GB, proteins such as Pavir.7KG134400 (SNF1-related protein kinase), Pavir.7KG108800 (LOX2), and Pavir.2KG476205 (Eukaryotic translation initiation factor 4G) were both upregulated in protein abundance and phosphorylation. Our comparative analysis revealed that switchgrass homologs of Arabidopsis defense regulators such as PR1, terpene synthase, papain cysteine protease, serine carboxypeptidase, and LOX2 were upregulated at both transcript and proteome levels. The patterns of protein localization of the DEPs and DPs were similar, with majority being nuclear localized, followed by plastid, membrane, and organelle membrane localization. Chloroplast localization of several DEPs and DPs is intriguing, since they produce many defense-related molecules, including JA and secondary messengers such as ROS. Similarly, the nucleus acts as the propagation hub of pathogen or herbivore-induced hormonal signaling pathways, leading to changes in gene expression and defense response. Also the secretory pathway participates in plant defense through delivery of defense proteins and defensive secondary metabolites to the extracellular space, and mediating localized callose deposition ${ }^{78}$. Therefore the extensive targeting of GB-feeding induced DPs and DEPs to the nucleus, chloroplast, and endomembrane system further reiterate the importance of these compartments to the switchgrass immune response.

Furthermore, phosphorylation of proteins like Pavir.9NG353828, a homolog to AtMYC2, may act as a key TF modulator of JA responses during plant defense ${ }^{79}$. Recently, rice (Oryza sativa) MYC2 was also reported to be an essential factor for JA-dependent production of sakuranetin ${ }^{80}$, a defense-related phytoalexin that accumulated in blast-infected rice leaves ${ }^{81}$. In the future, studies on the function of specific proteins found in this study, such as Pavir.9NG353828 ( $P v$ MYC2), will be helpful to explore the mechanisms of host resistance to GB and other aphid pests of switchgrass.

\section{Materials and methods}

Plant material and treatments. Plants of switchgrass cultivar Summer were grown from seed in Containers (Ray Leach SC10; Stuewe \& Sons, Inc, Tangent, OR) to the L2 stage ${ }^{82}$, under 400-W high intensity lamps with a 16:8 (light:dark) photoperiod and $23 \pm 4{ }^{\circ} \mathrm{C}$ temperature in a greenhouse $\mathrm{e}^{1}$. Fifty plants were randomly selected for this experiment. Plants were arranged in a $2 \times 4$ factorial design, which had two treatments (aphid infested and uninfested plants). Ten days post infestation (DPI) time point for leaf sample collection was selected based on previous transcriptomic data showing maximal upregulation of defense pathways $10 \mathrm{DPI}^{3}$. Each plant was infested with $10 \mathrm{~GB}$ (biotype I) at day 0 , after which each plant was caged with tubular plastic cages with vents covered with organdy fabric to restrict the aphid movement on the infested plants. Control plants (aphid uninfested) were similarly caged. Both aphid infested and control plants were kept in the greenhouse conditions described above for 10 days before leaf samples were taken. Aphids were removed from the leaves before sample collection. Leaves were flash frozen under liquid $\mathrm{N}_{2}$ conditions, ground to a fine powder using a mortar and pestle and were stored at $-80^{\circ} \mathrm{C}$, until analyzed.

Protein extraction and digestion. Total protein was extracted from four biological replicates of two treatments: C10 (controls) and I10 (infested). Protein extraction from the ground leaf tissues were done as described previously by Alvarez et al. ${ }^{83}$. The protein pellet was briefly air-dried, then redissolved in a solution containing $7 \mathrm{M}$ urea, $2 \mathrm{M}$ thiourea, $5 \mathrm{mM}$ DTT, containing 1x PhosSTOP phosphatase inhibitor (Roche, Basel, Switzerland) and 1x cOmplete, EDTA-free Protease Inhibitor Cocktail (Roche, Mannheim, Germany). Protein amounts were determined as previously described ${ }^{84} .800 \mu \mathrm{g}$ of total protein from each sample was reduced and alkylated as previously described in Alvarez et al. ${ }^{85}$. Samples were diluted tenfold and trypsin digestion carried out for $24 \mathrm{~h}$ at a ratio of 1:50 enzyme: substrate (E:S). A further aliquot of trypsin (1:50 E:S) was added and digestion carried out for a further $3 \mathrm{~h}$. Digests were acidified with 20\% TFA to $\mathrm{pH} 3$, then desalted using $50 \mathrm{mg}$ Sep-Pak C18 reverse-phase SPE columns (Waters Corp, Milford, MA). A portion was set aside for analysis of the unenriched sample.

Phosphoenrichment. Approximately, $0.75 \mathrm{mg}$ of digested, desalted, dry peptide was dissolved in $2 \mathrm{M}$ lactic acid, $60 \%$ acetonitrile to $3 \mathrm{mg} / \mathrm{mL}$ and shaken violently with $\mathrm{TiO}_{2}$ beads (Titansphere, $5 \mu \mathrm{m}$, GL Sciences, Tokyo, Japan) in a ratio of $1: 4$ sample:beads $(\mathrm{w} / \mathrm{w})$ for $1 \mathrm{~h}$ at $24^{\circ} \mathrm{C}$ as described previously ${ }^{86}$. The suspended beads were then placed into a $200 \mu \mathrm{L}$ tip (Eppendorf, Hauppauge, NY) plugged with 2 layers of $3 \mathrm{M}$ C8 Empore membrane (Thermo Fisher Scientific, USA). $100 \mu \mathrm{L}$ of the same solution was spun through the beads at $3,000 \times g$ three times. The beads were then further washed with $3 \times 100 \mu \mathrm{L}$ of $80 \%$ acetonitrile at 3,000 $\times$ g. Phosphopeptides were eluted into $1.5 \mathrm{~mL}$ Lo-Bind tubes (Eppendorf, Hauppauge, NY) by $2 \times 100 \mu \mathrm{L}$ additions of ammonium hydroxide $(5 \% \mathrm{v} / \mathrm{v})$ at $1,000 \times g$, frozen and immediately lyophilized. A further elution with $2 \times 100 \mu \mathrm{L}$ pyrrolidine $(5 \% \mathrm{v} / \mathrm{v})$ at $1,000 \times g$ was performed, and this pooled eluate was frozen and immediately lyophilized. Both eluates were combined and analyzed by LC-MS/MS. 
LC-MS/MS analysis of the proteome and phosphoproteome. The eight proteomic samples and the eight samples enriched for phosphopeptides were analysed by LC-MS/MS on an RSLCnano system (Thermo Fisher Scientific, USA) coupled to a Q-Exactive HF mass spectrometer (Thermo Fisher Scientific, USA). The samples were first injected onto a cartridge trap column (PepMap 100, C18, $0.3 \times 5 \mathrm{~mm}$, Thermo Fisher Scientific, USA) for $3.3 \mathrm{~min}$ at a flow rate of $5 \mu \mathrm{L} / \mathrm{min}, 2 \%$ acetonitrile, $0.1 \%$ formic acid before switching in line with the main column. Separation was performed on a C18 nano column (ACQUITY UPLC M-class, Peptide CSH 130A, $1.7 \mu \mathrm{m} 75 \mu \mathrm{m} \times 250 \mathrm{~mm}$, Waters Corp, Milford, MA) at $260 \mathrm{~nL} / \mathrm{min}$ with a linear gradient from $5-35 \%$ over $96 \mathrm{~min}$. The LC mobile phases were as follow: A contained $0.1 \%(\mathrm{v} / \mathrm{v})$ formic acid in water and B contained $0.1 \%$ $(\mathrm{v} / \mathrm{v})$ formic acid in $80 \%(\mathrm{v} / \mathrm{v})$ acetonitrile. Mass spectra for the eluted peptides were acquired on a Q Exactive HF mass spectrometer in data-dependent mode using a mass range of $m / z$ 375-1,500, resolution 120,000, AGC target $3 \times 10^{6}$, maximum injection time $60 \mathrm{~ms}$ for the MS1 peptide measurements. Data-dependent MS2 spectra were acquired by HCD as a Top20 experiment with a normalized collision energy (NCE) set at 28\%, AGC target set to $1 \times 10^{5}, 15,000$ resolution, intensity threshold $1 \times 10^{4}$ and a maximum injection time of 250 ms. Dynamic exclusion was set at $20 \mathrm{~s}$ to help capture phospho isomers and the isolation window set to $1.6 \mathrm{~m} / z$.

Data analysis. Data were analyzed in Proteome Discoverer 2.2 software (Thermo Fisher Scientific, USA). Three different database search tools were used, Mascot 2.6.2, MS Amanda 2.0 and SeQuest HT. The databases searched were the common contaminants database cRAP (116 entries, www.theGPM.org) and the Pvi5 (79,335 entries, www.phytozome.org). For the proteomics experiment, methionine oxidation, protein N-terminal and lysine acetylation, methylation, dimethylation and trimethylation of arginine and lysine were set as variable modifications, whilst Cys carbamidomethylation was specified as a fixed modification. For the phosphoproteomics experiment, methionine oxidation, protein N-terminal and lysine acetylation, and Ser/Thr and Tyr phosphorylation were set as variable modifications, whilst Cys carbamidomethylation was specified as a fixed modification. The search included a maximum of two trypsin missed cleavages with the precursor mass tolerance set to $10 \mathrm{ppm}$ and the fragment mass tolerance to $0.02 \mathrm{Da}$, respectively. Peptide validation were done by Percolator with a 0.01 posterior error probability (PEP) threshold. The data were searched using a decoy database to set the false discovery rate to $1 \%$ (high confidence). The localization probabilities of the PTMs were obtained using ptmRS ${ }^{87}$. The peptides were quantified using the precursor abundance based on intensity. The peak abundance was normalized for differences in sample loading using total peptide amount where the peptide group abundances were summed for each sample and the maximum sum across all runs was determined. The normalization factor used was the factor of the sum of the sample and the maximum sum in all files. The protein ratios, expressed as $\log _{2}$ fold change (Infected/Control), or $\log _{2} \mathrm{FC}$ (I10/C10), were calculated using summed of the peptides abundance for each sample and replicate separately. The geometric median from the four replicates was used to calculate the protein ratios. To compensate for missing values in some of the replicates, the replicatebased resampling imputation mode was selected. The significance of differential expression was tested using an ANOVA test, which provides adjusted p-values using the Benjamini-Hochberg method for all the calculated ratios, based on the summed abundances from the four replicates. For the phosphoproteomic analysis, data was filtered further to remove phosphopeptides with phosphosites not confidently localized (score $<95 \%$ according to ptmRS). The quantitative analysis was done at the phosphopeptide level and not at the phosphoprotein level for a better representation of the phosphorylation abundance change for each protein. Phosphosites identified to more than one gene ID were counted several times when reporting the proteins differentially phosphorylated.

Integration of proteomic, transcriptomic and phosphopeptides data. The integration between the transcriptomic, proteomic and phosphopeptide data is presented in the Table S7. The transcriptome data used in this study was previously published ${ }^{3}$. We retained only the information collected at 10 days for both aphid infested and uninfested controls. Genes with the $\mathrm{FC}>|2|$ and adjusted $p$ value $<0.05$ were identified as differentially expressed genes (DEGs). For the protein abundance and phosphoprotein, differentially expressed proteins (DEPs) and differentially phosphorylated-sites (DPs) were identified by adjusted $p$ value $<0.05$.

Functional annotation. The GOBU package was used for enrichment calculations ${ }^{26}$. The full set of switchgrass gene annotation was used as the reference comparison set against down or upregulated DEPs. The $p$ values were calculated using Fisher's exact test and corrected for multiple testing with FDR method using the R module called 'p-adjust'.

\section{Data availability}

Proteomic data have been deposited under https://proteomecentral.proteomexchange.org/cgi/GetDataset. The temporary numbers are 1-20200410-130072 for the proteomics study and 1-20200410-77144 for the phosphoproteomics study. The RNA-Seq datasets utilized in this study are available in the SRA repository, Accession number SRX1600826. Other datasets generated and/or analyzed during the current study are available from the corresponding authors on reasonable request.

Received: 12 April 2020; Accepted: 19 August 2020

Published online: 09 September 2020

\section{References}

1. Koch, K. G. et al. Evaluation of tetraploid switchgrass (Poales: Poaceae) populations for host suitability and differential resistance to four cereal aphids. J. Econ. Entomol. 107, 424-431 (2014). 
2. Koch, K. G. et al. Evaluation of greenbug and yellow sugarcane aphid feeding behavior on resistant and susceptible switchgrass cultivars. BioEnergy Res. 11, 480-490 (2018).

3. Donze-Reiner, T. et al. Transcriptional analysis of defense mechanisms in upland tetraploid switchgrass to greenbugs. BMC Plant Biol. 17, 46-46 (2017).

4. Smith, M. Plant Resistance to Arthropods: Molecular and Conventional Approaches (Springer, Dordrecht, 2005).

5. Painter, R. H. Insect Resistance in Crop Plants (Macmillan, New York, 1951).

6. Smith, C. M. \& Chuang, W.-P. Plant resistance to aphid feeding: behavioral, physiological, genetic and molecular cues regulate aphid host selection and feeding. Pest Manag. Sci. 70, 528-540 (2014).

7. Koch, K. G. et al. Characterization of greenbug feeding behavior and aphid (Hemiptera: Aphididae) host preference in relation to resistant and susceptible tetraploid switchgrass populations. BioEnergy Res. 8, 165-174 (2015).

8. Koch, K. G., Bradshaw, J. D., Heng-Moss, T. M. \& Sarath, G. Categories of resistance to greenbug and yellow sugarcane aphid (Hemiptera: Aphididae) in three tetraploid switchgrass populations. BioEnergy Res. 7, 909-918 (2014).

9. Foyer, C. H., Verrall, S. R. \& Hancock, R. D. Systematic analysis of phloem-feeding insect-induced transcriptional reprogramming in Arabidopsis highlights common features and reveals distinct responses to specialist and generalist insects. J. Exp. Bot. 66, 495-512 (2014).

10. Kerchev, P. I., Fenton, B., Foyer, C. H. \& Hancock, R. D. Plant responses to insect herbivory: interactions between photosynthesis, reactive oxygen species and hormonal signalling pathways. Plant Cell Environ. 35, 441-453 (2012).

11. Koch, K. G., Chapman, K., Louis, J., Heng-Moss, T. \& Sarath, G. Plant tolerance: a unique approach to control hemipteran pests. Front. Plant Sci. 7, 1363-1363 (2016).

12. Baxter, A., Mittler, R. \& Suzuki, N. ROS as key players in plant stress signalling. J. Exp. Bot. 65, 1229-1240 (2013).

13. Heng-Moss, T., Macedo, T., Franzen-Castle, L., Baxendale, F. \& Higley, L. Physiological responses of resistant and susceptible buffalograsses to Blissus occiduus (Hemiptera: Blissidae) feeding. J. Econ. Entomol. 99, 222-228 (2006).

14. Ramm, C. et al. Transcriptional profiling of resistant and susceptible buffalograsses in response to Blissus occiduus (Hemiptera: Blissidae) feeding. J. Econ. Entomol. 108, 1354-1362 (2015).

15. Saathoff, A. et al. Towards uncovering the roles of switchgrass peroxidases in plant processes. Front. Plant Sci. 4, 202 (2013).

16. Muneer, S., Jeong, H. K., Park, Y. G. \& Jeong, B. R. Proteomic analysis of aphid-resistant and -sensitive rose (Rosa hybrida) cultivars at two developmental stages. Proteomes 6, 25 (2018).

17. Truong, D.-H. et al. Proteomic analysis of Arabidopsis thaliana (L.) Heynh responses to a generalist sucking pest (Myzus persicae Sulzer). Plant Biol. 17, 1210-1217 (2015).

18. Duceppe, M.-O., Cloutier, C. \& Michaud, D. Wounding, insect chewing and phloem sap feeding differentially alter the leaf proteome of potato, Solanum tuberosum L.. Proteome Sci 10, 73-73 (2012).

19. Zogli, P., Pingault, L., Grover, S. Louis, J. Ento(o)mics: the intersection of "omic" approaches to decipher plant defense against sap-sucking insect pests. Curr. Opin. Plant Biol. 56, 153-161 (2020)

20. Guan, W. et al. Proteomic analysis shows that stress response proteins are significantly up-regulated in resistant diploid wheat (Triticum monococcum) in response to attack by the grain aphid (Sitobion avenae). Mol. Breed. 35, 57-57 (2015).

21. Coppola, V. et al. Transcriptomic and proteomic analysis of a compatible tomato-aphid interaction reveals a predominant salicylic acid-dependent plant response. BMC Genom. 14, 515-515 (2013).

22. Wu, X. et al. Proteomic analysis by iTRAQ-PRM provides integrated insight into mechanisms of resistance in pepper to Bemisia tabaci (Gennadius). BMC Plant Biol. 19, 270 (2019).

23. Zhang, X. et al. Proteomic analysis of the rice (Oryza officinalis) provides clues on molecular tagging of proteins for brown planthopper resistance. BMC Plant Biol. 19, 30-30 (2019).

24. Castano-Duque, L. \& Luthe, D. S. Protein networks reveal organ-specific defense strategies in maize in response to an aboveground herbivore. Arthropod Plant Interact. 12, 147-175 (2018).

25. Lee, J. \& Koh, H.-J. A label-free quantitative shotgun proteomics analysis of rice grain development. Proteome Sci. 9, 61 (2011).

26. Lin, W.-D., Chen, Y.-C., Ho, J.-M. \& Hsiao, C.-D. GOBU: toward an integration interface for biological objects. J. Inf. Sci. Eng. 22, 19-29 (2006).

27. Sharma, E., Anand, G. \& Kapoor, R. Terpenoids in plant and arbuscular mycorrhiza-reinforced defence against herbivorous insects. Ann. Bot. 119, 791-801 (2017).

28. Pré, M. et al. The AP2/ERF domain transcription factor ORA59 integrates jasmonic acid and ethylene signals in plant defense. Plant Physiol. 147, 1347 (2008).

29. Choi, H. W. et al. A role for a menthone reductase in resistance against microbial pathogens in plants. Plant Physiol. 148, 383 (2008).

30. Alazem, M., Lin, K.-Y. \& Lin, N.-S. The abscisic acid pathway has multifaceted effects on the accumulation of bamboo mosaic virus. MPMI 27, 177-189 (2013)

31. Park, C.-J., Caddell, D. F. \& Ronald, P. C. Protein phosphorylation in plant immunity: insights into the regulation of pattern recognition receptor-mediated signaling. Front. Plant Sci. 3, 177-177 (2012).

32. Jagodzik, P., Tajdel-Zielinska, M., Ciesla, A., Marczak, M. \& Ludwikow, A. Mitogen-activated protein kinase cascades in plant hormone signaling. Front. Plant Sci. 9, 1387 (2018).

33. Muchlinski, A. et al. Biosynthesis and emission of stress-induced volatile terpenes in roots and leaves of switchgrass (Panicum virgatum L.). Front. Plant Sci. 10, 1144 (2019).

34. Chen, J.-H. et al. Drought and salt stress tolerance of an Arabidopsis glutathione $S$-transferase U17 knockout mutant are attributed to the combined effect of glutathione and abscisic acid. Plant Physiol. 158, 340 (2012).

35. Wang, X. Protein and proteome atlas for plants under stresses: new highlights and ways for integrated omics in post-genomics era. Int. J. Mol. Sci. 20, 5222 (2019).

36. Liu, Y. et al. Proteomics: a powerful tool to study plant responses to biotic stress. Plant Methods 15, 135 (2019).

37. Dong, Y. et al. Comparative proteomic analysis of susceptible and resistant rice plants during early infestation by small brown planthopper. Front. Plant Sci. 8, 1744-1744 (2017).

38. Ulappa, A. C. et al. Plant protein and secondary metabolites influence diet selection in a mammalian specialist herbivore. J. Mammal 95, 834-842 (2014).

39. Zaynab, M. et al. Role of secondary metabolites in plant defense against pathogens. Microb. Pathog. 124, 198-202 (2018).

40. Hansen, B. G. et al. A novel 2-oxoacid-dependent dioxygenase involved in the formation of the goiterogenic 2-hydroxybut-3-enyl glucosinolate and generalist insect resistance in Arabidopsis. Plant Physiol. 148, 2096 (2008).

41. Ford, K. A. et al. Neonicotinoid insecticides induce salicylate-associated plant defense responses. Proc. Natl. Acad. Sci. USA 107, $17527-17532(2010)$

42. Tzin, V. et al. Dynamic maize responses to aphid feeding are revealed by a time series of transcriptomic and metabolomic assays. Plant Physiol. 169, 1727-1743 (2015).

43. Zhou, S., Richter, A. \& Jander, G. Beyond defense: multiple functions of benzoxazinoids in maize metabolism. Plant Cell Physiol. 59, 1528-1537 (2018).

44. Yang, C. et al. Lignin metabolism involves Botrytis cinerea BcGs1-induced defense response in tomato. BMC Plant Biol. 18, 103 (2018). 
45. Glauser, G. et al. Spatial and temporal dynamics of jasmonate synthesis and accumulation in Arabidopsis in response to wounding. J. Biol. Chem. 283, 16400-16407 (2008).

46. Zhang, L., Zhang, F., Melotto, M., Yao, J. \& He, S. Y. Jasmonate signaling and manipulation by pathogens and insects. J. Exp. Bot. 68, 1371-1385 (2017).

47. Thivierge, K. et al. Caterpillar- and salivary-specific modification of plant proteins. J. Proteome Res. 9, 5887-5895 (2010).

48. Nalam, V. J., Keeretaweep, J., Sarowar, S. \& Shah, J. Root-derived oxylipins promote green peach aphid performance on Arabidopsis foliage. Plant Cell 24, 1643-1653 (2012).

49. Maffei, M. E., Mithöfer, A. \& Boland, W. Before gene expression: early events in plant-insect interaction. Trends Plant Sci. 12, $310-316(2007)$.

50. Vincent, T. R. et al. Interplay of plasma membrane and vacuolar ion channels, together with BAK1, elicits rapid cytosolic calcium elevations in Arabidopsis during aphid feeding. Plant Cell 29, 1460-1479 (2017).

51. Sun, M., Voorrips, R. E. \& Vosman, B. Aphid populations showing differential levels of virulence on Capsicum accessions. Insect Sci. 27, 336-348 (2020).

52. Kehr, J. Phloem sap proteins: their identities and potential roles in the interaction between plants and phloem-feeding insects. J. Exp. Bot. 57, 767-774 (2006).

53. Peng, H.-C. \& Walker, G. P. Sieve element occlusion provides resistance against Aphis gossypii in TGR-1551 melons. Insect Sci. 27, 33-48 (2020).

54. Sun, M., Voorrips, R. E., Steenhuis-Broers, G., Van't Westende, W. \& Vosman, B. Reduced phloem uptake of Myzus persicae on an aphid resistant pepper accession. BMC Plant Biol. 18, 138-138 (2018).

55. Moloi, M. J. \& van der Westhuizen, A. J. The reactive oxygen species are involved in resistance responses of wheat to the Russian wheat aphid. J. Plant Physiol. 163, 1118-1125 (2006).

56. Park, S.-J., Huang, Y. \& Ayoubi, P. Identification of expression profiles of sorghum genes in response to greenbug phloem-feeding using cDNA subtraction and microarray analysis. Planta 223, 932-947 (2006).

57. Torres, M. A., Dangl, J. L. \& Jones, J. D. G. Arabidopsis gp91 ${ }^{\text {phox }}$ homologues AtrbohD and AtrbohF are required for accumulation of reactive oxygen intermediates in the plant defense response. Proc. Natl. Acad. Sci. USA 99, 517-522 (2002).

58. Bindschedler, L. V. et al. Peroxidase-dependent apoplastic oxidative burst in Arabidopsis required for pathogen resistance. Plant J. 47, 851-863 (2006).

59. Hu, X. et al. Overexpression of a gene encoding hydrogen peroxide-generating oxalate oxidase evokes defense responses in sunflower. Plant Physiol. 133, 170 (2003).

60. Mhamdi, A. et al. Catalase function in plants: a focus on Arabidopsis mutants as stress-mimic models. J. Exp. Bot. 61, 4197-4220 (2010).

61. de Carvalho-Oliveira, R. A. et al. Analysis of Arabidopsis thaliana redox gene network indicates evolutionary expansion of class iii peroxidase in plants. Sci. Rep. 9, 15741 (2019).

62. Dreyer, B. H. \& Schippers, J. H. M. Copper-zinc superoxide dismutases in plants: evolution, enzymatic properties, and beyond. In Annual Plant Reviews online 933-968 (American Cancer Society, 2019). https://doi.org/10.1002/9781119312994.apr0705.

63. Noctor, G. \& Foyer, C. H. Ascorbate and glutathione: keeping active oxygen under control. Annu. Rev. Plant Biol. 49, 249-279 (1998).

64. Almagro, L. et al. Class III peroxidases in plant defence reactions. J. Exp. Bot. 60, 377-390 (2009).

65. Ye, Z. et al. Comparative proteomics of root apex and root elongation zones provides insights into molecular mechanisms for drought stress and recovery adjustment in switchgrass. Proteomes 8, 3 (2020).

66. Gaquerel, E., Steppuhn, A. \& Baldwin, I. T. Nicotiana attenuata $\alpha$-DIOXYGENASE1 through its production of 2-hydroxylinolenic acid is required for intact plant defense expression against attack from Manduca sexta larvae. New Phytol. 196, 574-585 (2012).

67. Avila, C. A., Arevalo-Soliz, L. M., Lorence, A. \& Goggin, F. L. Expression of a-DIOXYGENASE 1 in tomato and Arabidopsis contributes to plant defenses against aphids. MPMI 26, 977-986 (2013).

68. Pan, X. et al. iTRAQ Protein profile analysis of tomato green-ripe mutant reveals new aspects critical for fruit ripening. J. Proteome Res. 13, 1979-1993 (2014).

69. Misas-Villamil, J. C., van der Hoorn, R. A. L. \& Doehlemann, G. Papain-like cysteine proteases as hubs in plant immunity. New Phytol. 212, 902-907 (2016).

70. Louis, J. et al. Ethylene contributes to maize insect resistance1-mediated maize defense against the phloem sap-sucking corn leaf aphid. Plant Physiol. 169, 313 (2015).

71. Lee, M. W., Jelenska, J. \& Greenberg, J. T. Arabidopsis proteins important for modulating defense responses to Pseudomonas syringae that secrete HopW1-1. Plant J. 54, 452-465 (2008).

72. Toruño, T. Y., Shen, M., Coaker, G. \& Mackey, D. Regulated disorder: posttranslational modifications control the RIN4 plant immune signaling hub. MPMI 32, 56-64 (2018).

73. Redditt, T. J. et al. AvrRpm1 functions as an ADP-ribosyl transferase to modify NOI domain-containing proteins, including Arabidopsis and soybean RPM1-interacting protein4. Plant Cell 31, 2664 (2019).

74. Wang, Z. et al. A family of NAI2-interacting proteins in the biogenesis of the ER body and related structures. Plant Physiol. 180, $212(2019)$.

75. Nakazaki, A. et al. Leaf endoplasmic reticulum bodies identified in Arabidopsis rosette leaves are involved in defense against herbivory. Plant Physiol. 179, 1515 (2019).

76. Nakano, R. T. et al. PYK10 myrosinase reveals a functional coordination between endoplasmic reticulum bodies and glucosinolates in Arabidopsis thaliana. Plant J. 89, 204-220 (2017).

77. Zhou, S., Lou, Y.-R., Tzin, V. \& Jander, G. Alteration of plant primary metabolism in response to insect herbivory. Plant Physiol. 169, 1488-1498 (2015).

78. Wang, W.-M., Liu, P.-Q., Xu, Y.-J. \& Xiao, S. Protein trafficking during plant innate immunity. J. Integr. Plant Biol. 58, 284-298 (2016).

79. Lorenzo, O., Chico, J. M., Sánchez-Serrano, J. J. \& Solano, R. JASMONATE-INSENSITIVE1 encodes a MYC transcription factor essential to discriminate between different jasmonate-regulated defense responses in Arabidopsis. Plant Cell 16, 1938-1950 (2004).

80. Ogawa, S. et al. OsMYC2, an essential factor for JA-inductive sakuranetin production in rice, interacts with MYC2-like proteins that enhance its transactivation ability. Sci. Rep. 7, 40175 (2017).

81. Hasegawa, M. et al. Analysis on blast fungus-responsive characters of a flavonoid phytoalexin sakuranetin; accumulation in infected rice leaves, antifungal activity and detoxification by fungus. Molecules 19, 11404 (2014).

82. Moore, K. et al. Describing and quantifying growth stages of perennial forage grasses. Agron. J. 83, 1073-1077 (1991).

83. Alvarez, S., Hicks, L. M. \& Pandey, S. ABA-dependent and-independent G-protein signaling in Arabidopsis roots revealed through an iTRAQ proteomics approach. J. Proteome Res. 10, 3107-3122 (2011).

84. Alvarez, S., Roy Choudhury, S. \& Pandey, S. Comparative quantitative proteomics analysis of the ABA response of roots of drought-sensitive and drought-tolerant wheat varieties identifies proteomic signatures of drought adaptability. J. Proteome Res. 13, 1688-1701 (2014).

85. Alvarez, S., Roy Choudhury, S., Sivagnanam, K., Hicks, L. M. \& Pandey, S. Quantitative proteomics analysis of Camelina sativa seeds overexpressing the AGG3 gene to identify the proteomic basis of increased yield and stress tolerance. J. Proteome Res. 14, 2606-2616 (2015). 
86. Kettenbach, A. N. \& Gerber, S. A. Rapid and reproducible single-stage phosphopeptide enrichment of complex peptide mixtures: Application to general and phosphotyrosine-specific phosphoproteomics experiments. Anal. Chem. 83, 7635-7644 (2011).

87. Taus, T. et al. Universal and confident phosphorylation site localization using phosphoRS. J. Proteome Res. 10, 5354-5362 (2011).

\title{
Acknowledgements
}

We thank Katherine Keller for laboratory help. This work was supported in part by Grants from USDA-NIFA Grant Number 2011-67009-30096 (GS, THM, JDB), and by the USDA-ARS CRIS Project 3042-21000-034-00D. The U.S. Department of Agriculture, Agricultural Research Service, is an equal opportunity/affirmative action employer and all agency services are available without discrimination. Mention of commercial products and organizations in this manuscript is solely to provide specific information. It does not constitute endorsement by USDA-ARS over other products and organizations not mentioned. The proteomic and phosphoproteomic analyses were done on the LC-QE-HF mass spectrometer funded by the Nebraska Research Initiative. Work in the JL laboratory was supported by US National Science Foundation CAREER Grant IOS-1845588.

\section{Author contributions}

K.G.K., N.A.P., T.H.M., J.D.B., and G.S. designed the study. K.G.K. and N.A.P. collected the tissues. S.A. and M.J.N. performed the proteomics and phosphoproteomics experiments and interpreted the data. P.Z., N.A.P., L.P., and P.T. contributed to reagents/materials and data analysis. P.Z., N.A.P., J.L. and G.S. co-wrote the draft of the paper. J.L. provided funding and data interpretation. All authors contributed to the writing and editing of the final manuscript.

\section{Competing interests}

The authors declare no competing interests.

\section{Additional information}

Supplementary information is available for this paper at https://doi.org/10.1038/s41598-020-71828-8.

Correspondence and requests for materials should be addressed to J.L. or G.S.

Reprints and permissions information is available at www.nature.com/reprints.

Publisher's note Springer Nature remains neutral with regard to jurisdictional claims in published maps and institutional affiliations.

\begin{abstract}
Open Access This article is licensed under a Creative Commons Attribution 4.0 International License, which permits use, sharing, adaptation, distribution and reproduction in any medium or format, as long as you give appropriate credit to the original author(s) and the source, provide a link to the Creative Commons licence, and indicate if changes were made. The images or other third party material in this article are included in the article's Creative Commons licence, unless indicated otherwise in a credit line to the material. If material is not included in the article's Creative Commons licence and your intended use is not permitted by statutory regulation or exceeds the permitted use, you will need to obtain permission directly from the copyright holder. To view a copy of this licence, visit http://creativecommons.org/licenses/by/4.0/.
\end{abstract}

(c) The Author(s) 2020 